\title{
Improvement on LEACH Protocol in Wireless Sensor Networks
}

\author{
Raju Dutta \\ Narula Institute of Technology \\ Dept. of Mathematics \\ Kolkata, West Bengal
}

\author{
Shishir Gupta \\ Indian School of Mines \\ Dept. of Applied Mathematics \\ Dhanbad, Jharkhand
}

\author{
Mukul K Das \\ Indian School of Mines \\ Dept. of Electronics \\ Engineering \\ Dhanbad, Jharkhand
}

\begin{abstract}
Wireless Sensor Networks (WSNs) have great potential to support several important wireless applications, including real-time multimedia communication, medical applications, surveillance using sensor networks, industrial applications, military surveillance and home networking applications. But there are two challenging issues (i) communication bandwidth and (ii) energy are very important to design wireless and mobile systems because these are very much limited in network environment. Therefore it requires intelligent communication and design techniques to increase bandwidth as well as energy efficient protocol. The most efficient routing protocol LEACH (Lower Energy Adaptive Clustering Hierarchy) in wireless sensor networks (WSNs) has been incorporated by Heinzelman et.al. LEACH uses the concept of dynamic clustering when sensor nodes are deploying randomly where number of cluster impact on the network. This paper describes the network quality that depends on different characteristics of data transmission as a Modification on LEACH protocol. In this paper, we discussed and explain the comparison of magnitude, phase, phase delay, group delay, amplitude of broadcasting and energy consumption respectively.
\end{abstract}

\section{Keywords}

Protocol; Modified LEACH protocol; lifetime; Wireless Sensor Network.

\section{INTRODUCTION}

In recent years, the wireless sensor networks have become one of the hot topic of area of research. Wireless communication has shown its numerous advantages over wired communication and has within the last decade become a regular mode of communication in people's everyday lives.

The list of potential uses for wireless sensor networks seems to be unending, with different applications areas such as security, medicine, industrial machinery monitoring, the military, agriculture and others. These networks are no longer limited to military applications but are used in a wide array of applications including habitat monitoring [1], industrial process monitoring [2], traffic control [3], [4], health care [5], etc. This paper [6] improves the current security mechanisms in wireless sensor networks as well as reducing power consumption. LEACH protocol provides an energy routing protocol. However, it doesn't cover the security problems. Alternatively, this paper aims to provide an improved secure and more energy efficient routing protocol called LS-LEACH (Lightweight Secure LEACH). Authentication algorithm is integrated to assure data integrity, authenticity and availability. Furthermore, this paper shows the improvement over LEACH protocol that makes it secure and how to make it more energy efficient to reduce the effect of the overhead energy consumption from the added security measures. In [7] cluster based routing in wireless sensor networks is studied precisely. Further, authors modify one of the most prominent wireless sensor network's routing protocol "LEACH" as modified LEACH (MODLEACH) by introducing efficient cluster head replacement scheme and dual transmitting power levels. Our modified LEACH, in comparison with LEACH out performs it using metrics of cluster head formation, through put and network life. Afterwards, hard and soft thresholds are implemented on modified LEACH (MODLEACH) that boasts the performance even more. In [8] an improved routing algorithm based on LEACH, known as ILEACH, is proposed in this paper. Firstly, the ILEACH employed the residual energy to form clustering, which can avoid the low energy node becoming a cluster head. Secondly, an energy function is proposed to balance the energy consumption among cluster heads. Finally, a data aggregation tree is constructed to transmit the data from the cluster heads to sink node. Wireless sensor networks (WSNs) consists sensors which communicate to sensors by multihop. Generally research is continuing on sensor network through two stages, at the beginning stage is primarily intended for node and the last stage is for network-level issues. The main research works in this stage involve the network layer and MAC layer protocol based on energy optimization, node localization technology, clock synchronization technology and data fusion technology [9].

As the power of the sensor node can not be increased then how the nodes can be efficiently use in the network so that system energy becomes the prime factor for designing routing protocol. In this paper, we proposed a new energy model in our protocol and compare several aspects with existing LEACH protocol.

\section{LEACH PROTOCOL}

A typical hierarchical routing protocol is LEACH Protocol which is self-adaptive and self-organized. LEACH protocol uses round as unit for the purpose of reducing energy costs and each round is made up with two phases such as cluster set-up stage and steady-state stage. The steady-state phase must be longer than set-up phase. Wireless sensor networks is a subset of ad-hoc network consists static or dynamic nodes for data transmission in the network by self organized way and multi hop way. The main activity of node is data centric and collecting information for the user [10]. It is seen that sensor node performing mainly three functions data acquisition, processing and transmission, which are related to wireless technology, modern technology, computer and communication technology. Here the sensors are used as a basic element of WSNs where sensors are collecting, processing and transmitting information. 


\subsection{Cluster Head selection algorithm in LEACH}

Two phases are in LEACH protocol which is (i) the cluster formation and (ii) data receiving and transmission phase and round as defined the time slot gap between two phases. In the cluster head selection phase sensor node generates a random number which lies within 0 and 1 , if that number is less than threshold value $\mathrm{T}(\mathrm{n})$ then in that round it selects that node to act as clusterhead, and acknowledge that node to the other neighbor [11]. The formula for $\mathrm{T}(\mathrm{n})$ is given below:

$$
T(n)= \begin{cases}\frac{p}{1-p^{*}\left(r \bmod \frac{1}{p}\right),} & \text { if } n \in G \\ 0 & \text { otherwise }\end{cases}
$$

$r$ indicates the present round, selection of a node to be a clusterhaed with probability, $p$ and those nodes are still not become clusterhead in previous ( $\mathrm{r}-1)$ round, will form another set $G$. We consider here $N$ as the total number of nodes in the network, the approximate number of cluster head to be considered as $k$, where $p=k / N$. Each node has opportunity to become a cluster head once by this algorithm, if one node become cluster head in a particular round, it will lose to become another time cluster head in other round.

\section{MODIFIED LEACH PROTOCOL}

\subsection{Our Energy Model}

In our protocol we consider energy for each round. We consider our energy model as given below:

$$
\text { Energy }=\text { Energy }+S(i) \cdot E^{*} \frac{(i-1)}{r \max -i}
$$

Where $\mathrm{i}$ stands for number of iteration, rmax is maximum number of rounds, Energy as our initial energy and s(i).E consider as energy level of each node after rounds.

\subsection{Simulation Parameter}

Simulation parameters are considered in this paper as:

1. Sensor nodes are randomly deployed and distributed in a square region;

2. Sensor nodes are homogeneous, nodes energy is limited. The node's location does not vary much even after deployed.

3. Base station location consider according to the user or center of the region.

4. Communicate to base station from sensor node in either single-hop or multi-hop.

5. The wireless transmitter, receiver and data aggregation power is according to LEACH protocol.

Set monitoring area according to the simulation, the number of nodes can varies according to the need, hopcount varies with nodes, base station can be considered according to the user. Specific parameters are shown in Table I.

\subsection{Analysis of Modified LEACH}

Energy consumption of the network under different number of rounds for LEACH and Modified LEACH protocol has been carried out and from simulated result it is observed using Eq. (1) our protocol reduces energy consumption than LEACH protocol, which has been shown in Fig. 2.

\subsubsection{Simulation of Modified LEACH}

In our simulation we consider 100 nodes randomly distribute within the square area of the $100 \mathrm{~m} * 100 \mathrm{~m}$, the base station is located in the centre of the region, the base station coordinates is $(50,50)$. It can be seen from the Fig. 1 that the nodes' are distributed randomly.

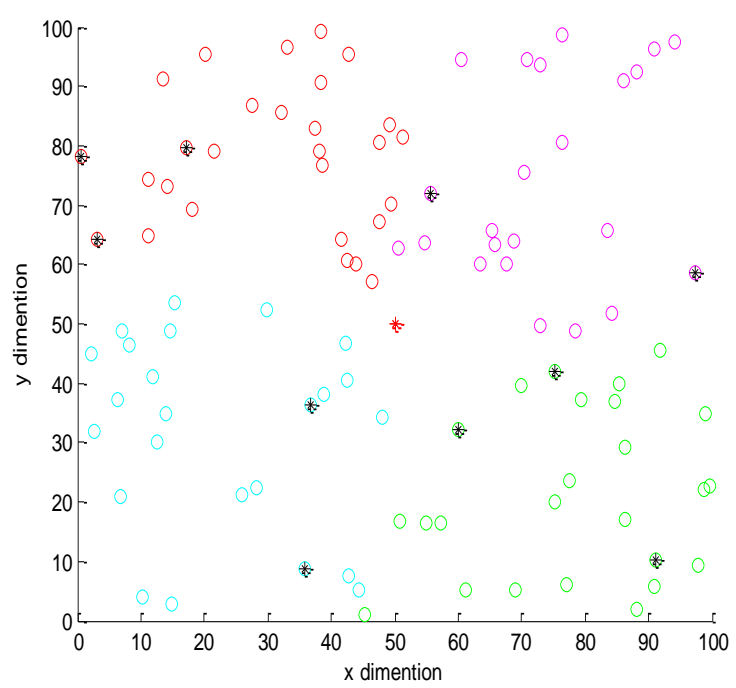

Fig 1: Randomly distributed nodes

In Fig. 1 cluster head are selecting from nodes and transmitting data to base station. Energy consumption is moe in LEACH than Modified LEACH. We Consider 100 nodes for simulation LEACH and Modified.

\subsubsection{Energy Consumption Simulation}

The comparison of energy consumption with node is shown in Fig. 2, where energy consumption by LEACH protocol is more than Modified LEACH protocol. Therefore the energy figures illustrates that Modified LEACH protocol reduces the energy consumption.

\subsubsection{Data Received by Base Station}

Fig. 8 shows the comparison between the amounts of data received by base station with time. There are effective broadcast by cluster head, because of the effective data transmission fail means retransmission is needed that will send more data to the base station. Here the simulation has been done upto 500s and cluster head stop sending data until the transmitted data reached at destination. Our protocol, Modified LEACH protocol under optimum number of cluster head can improve efficiency of the wireless sensor networks.

\section{SIMULATION RESULT}

LEACH uses broadcasting with multi-hop delivery to send the packets to the whole network which will follow all possible routes to its destination until the massage sent successfully. In Fig. 3 we comparison the magnitude of both the transmission between our protocol and LEACH protocol, where the magnitude by our proposed protocol is lower than 
LEACH protocol, indicates to transmit data from sensor node to cluster head and to base station will consume minimum energy. In Fig. 4 phase recognition is more or less linear in our proposed protocol implies data transmission is smooth and will consume less energy to send data than LEACH. In Fig5 and Fig. 6 describe phase delay and group delay between our protocol and LEACH and seen both the delay in our protocol is much more linear than LEACH. Fig. 7 implies amplitude of transmission of Modified LEACH is not varying abruptly compare to LEACH protocol. Table I shows the transmission parameter values used for the simulation of both the routing protocols and it is seen that Modified LEACH will extend lifetime of the network than LEACH.

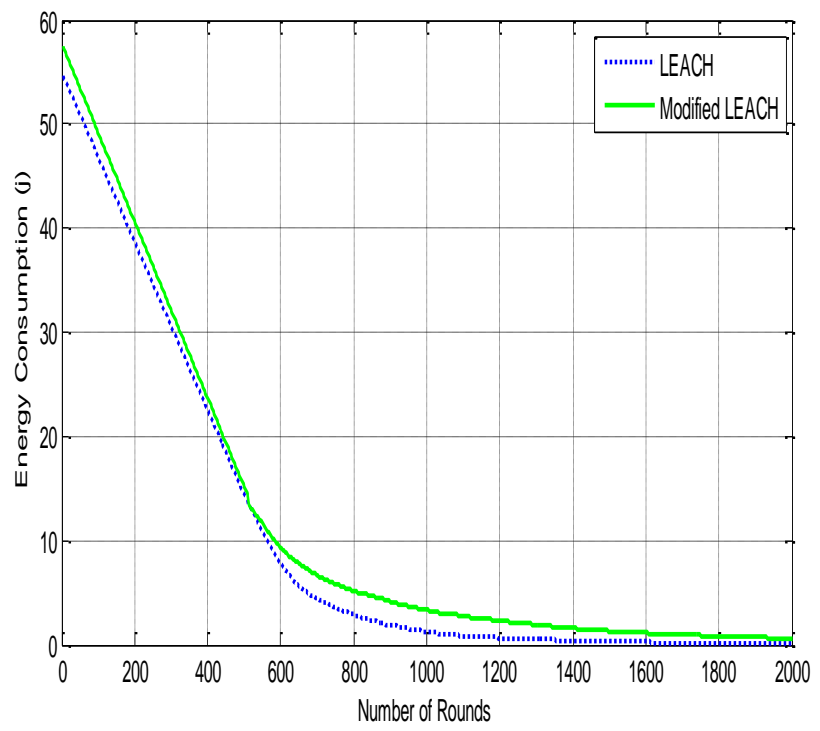

Fig 2: Comparison of energy consumption between our protocol and LEACH

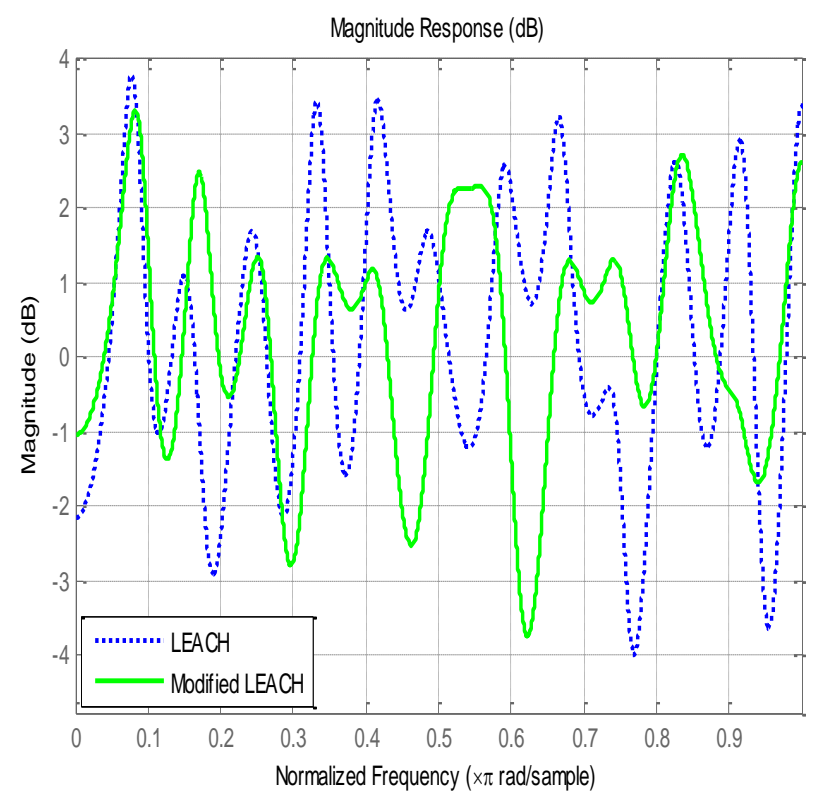

Fig 3: Comparison of Magnitude of data transmission between our protocol and LEACH

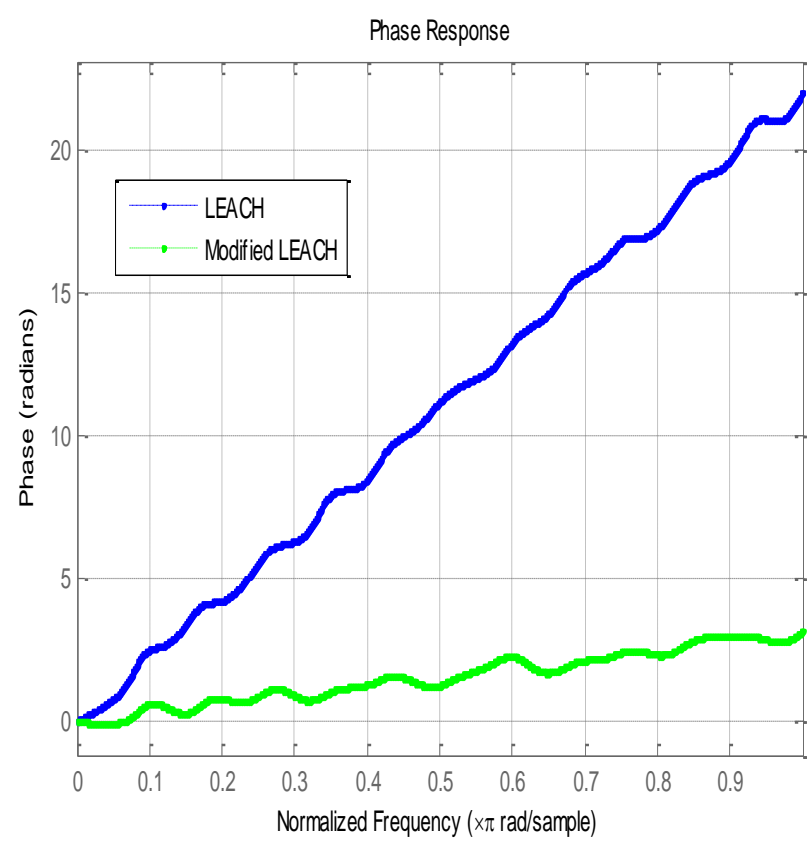

Fig 4: Phase recognition for Modified LEACH and LEACH

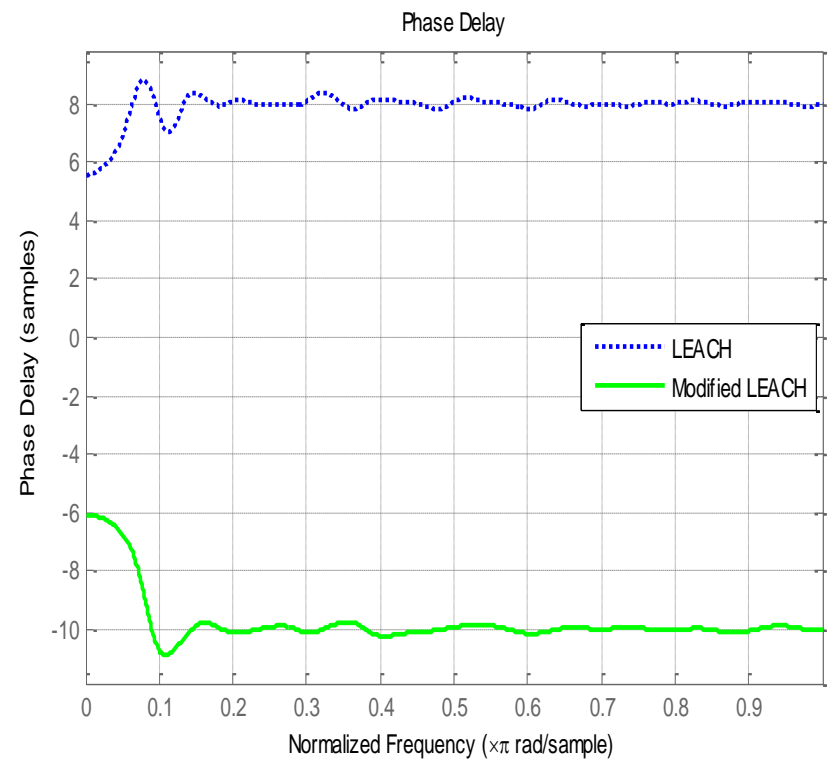

Fig 5: Phase delay between our protocol and LEACH 


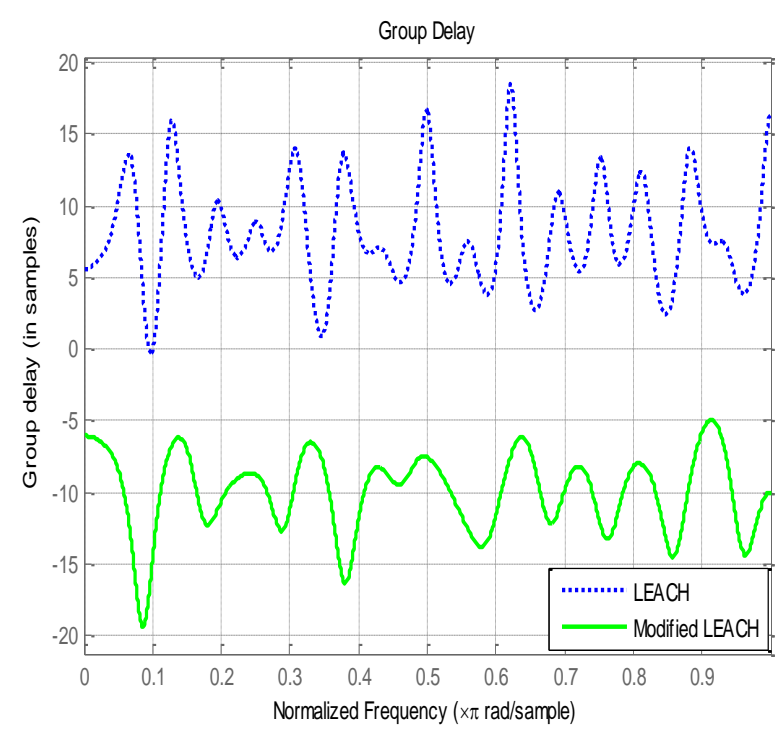

Fig 6: Group delay between the protocols

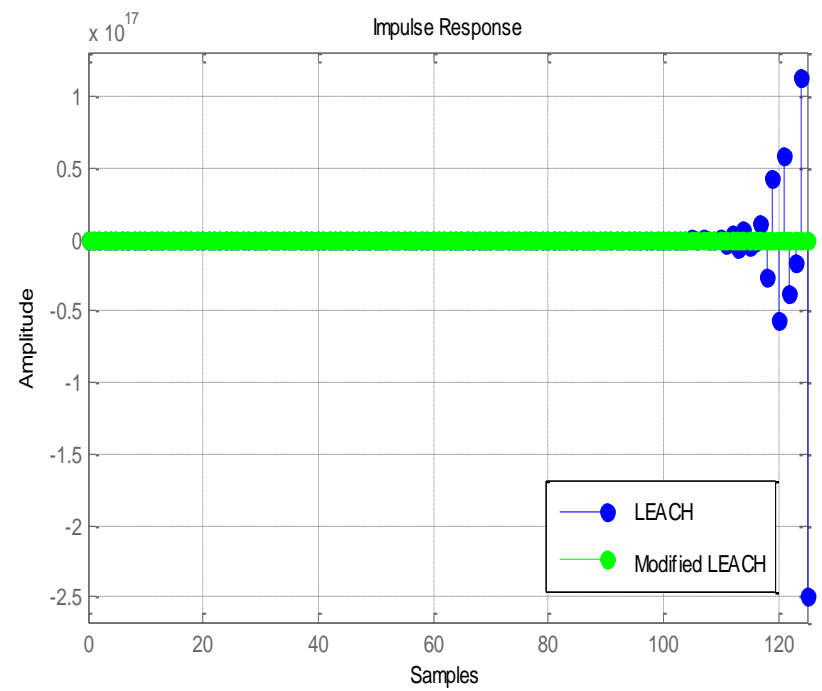

Fig 7: Amplitude of transmission of Modified LEACH and LEACH

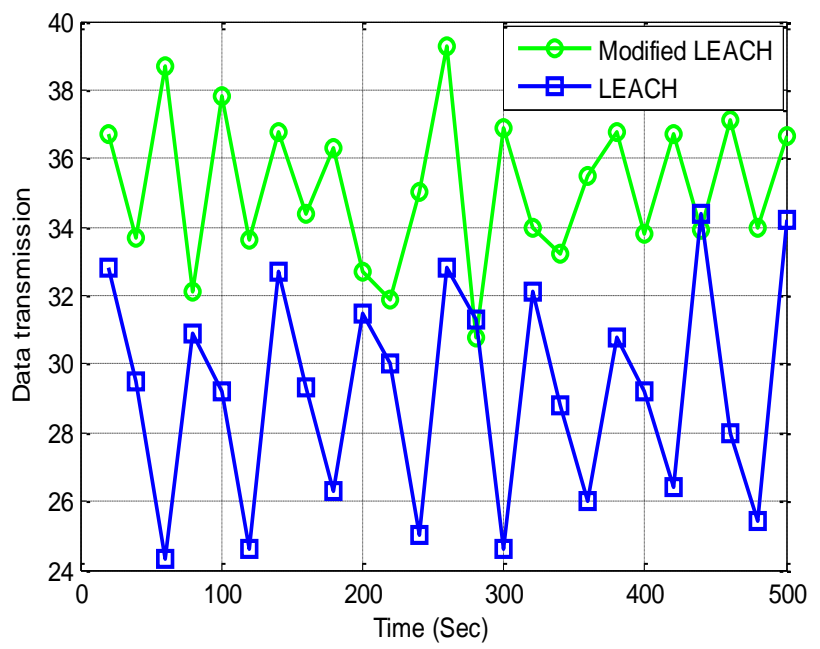

Fig 8: Data transmission of Modified LEACH and LEACH protocol

\begin{tabular}{|lcr|}
\hline Parameters & Notations & Value \\
\hline Number of nodes & $\mathrm{N}$ & 100 \\
Base Station location & $\left(x_{i}, y_{i}\right)$ & $(50,50)$ \\
The initial node energy & $E_{0}$ & $0.5 \mathrm{~J}$ \\
Energy consumed by the amplifier to transmit & \\
at shortest distance & $E_{a m p}$ & $120 \mathrm{pJ} / \mathrm{bit} / \mathrm{m}^{2}$ \\
Energy consumed by the amplifier to & \\
transmit at longest distance & $E_{f s}$ & $10 \mathrm{pJ} / \mathrm{bit} / \mathrm{m}^{2}$ \\
Energy consumed in the electronics circuit to \\
transmit or receive the signal & $E_{\text {elec }}$ & $70 \mathrm{pJ} / \mathrm{bit}$ \\
Data packet & $K$ & $4096 \mathrm{bits} / \mathrm{frame}$ \\
Data aggregation energy & $E_{d a}$ & $5 \mathrm{~nJ} / \mathrm{bit} / \mathrm{report}$ \\
The cluster probability & \multicolumn{3}{l}{} \\
of Modified LEACH & $\mathrm{p}$ & 0.1 \\
The Sensing area & $\mathrm{M} \times \mathrm{M}$ & $\mathbf{1 0 0 M} \times 100 \mathrm{M}$ \\
Simulation time & $\mathrm{T}$ & $3 \mathrm{hours}$ \\
\hline
\end{tabular}

Table 1: Transmission Parameters Value used for simulation

\section{PERFORMANCE ANALYSIS}

Heuristic simulation carried out by using MATLAB for both LEACH and Modified LEACH. In Fig. 2 compares energy consumption with increasing on nodes in the network and shows Modified LEACH prolong system lifetime. Fig. 4 explains node broadcast with time is increasing than LEACH protocol. The result in Fig. 5 shows better perform of modified LEACH indicates for long run Modified LEACH will be much more applicable than LEACH.

\section{CONCLUSION}

LEACH and Modified LEACH protocols were implemented in TinyOS with some degree of success. The basic evaluation of these protocols was carried out and different phase comparison has been made and showed by different graphs. During the design and implementation of the protocols it was clear that performance gains by Modified LEACH better than LEACH. The implemented protocols might prove to be more successful when used for routing packets in sensor networks.

\section{REFERENCES}

[1] Mainwaring, A, Culler, D, Polastre, J, Szewczyk, R and Anderson, J. 2002. "Wireless Sensor Networks for Habitat Monitoring". In Proceedings of the 1st ACM International Workshop on Wireless Sensor Networks and Applications, pp.88-97.

[2] Antoniou, M, Boon, M. C., Green, P. N., Green, P.R., and T.A. York, T.A. 2009. "Wireless Sensor Networks for Industrial Processes". In Sensors Applications Symposium, pp.13-18.

[3] Liu J and Fang, Y. 2006. "Urban Traffic Control System Based on Wireless Sensor Networks". IEEE International Conference on in Information Acquisition, pp.295-300.

[4] Zhang, H, Pan, C, Yang, J, Dong, H, Qin, Y and Jia, L. 2010. "SN-UTIA: A Sensor Network for Urban Traffic Information Acquisition". In Intelligent Vehicles Symposium (IV), pp. $566-571$. 
[5] Milenkovi, A, Otto, C and Jovanov, E. 2006. "Wireless Sensor Networks for Personal Health Monitoring: Issues and an Implementation". Computer Communications (Special issue: Wireless Sensor Networks: Performance, Reliability, Security, and Beyond, 29, pp.2521-2533.

[6] Alshowkan, M, Elleithy, K, AlHassan, H. 2013.'LSLEACH: A New Secure and Energy Efficient Routing Protocol for Wireless Sensor Networks" in 17th IEEE/ACM International Symposium on Distributed Simulation and Real Time Applications, pp.215-220.

[7] Mahmood, D, Javaid, N, Mahmood, S, Qureshi, S, Memon, A.M, Zaman, T. 2013. "MODLEACH: A Variant of LEACH for WSNs" Eighth International Conference on Broadband, Wireless Computing, Communication and Applications, pp158-163.
[8] Jing, Y, Zetao, L, Yi, L. 2013. "An Improved Routing Algorithm Based on LEACH for Wireless Sensor Networks", 25th Chinese Control and Decision Conference (CCDC), pp-3716-3720.

[9] Roseline, R. A. and Sumathi, P. 2011. "Energy Efficient Routing Protocol and Algorithms for Wireless Sensor Networks-A Survey". Global Journal of Computer Science and Technology, 11(21).

[10] Xun-xue, C. 2009. "Wireless Sensor Network Brief Tutorial", Qing Hua University Press,Beijing.

[11] Mei-hong, H., Cheng-qian, X. and Dong-liang, Z. 2009. "The simulation and analysis of LEACH protocol Based on NS2", Electronic Measurement Technology, vol.1, pp.40-42. 